\title{
Article
}

\section{Effectiveness of Psychological Interventions to Improve the Mental Well- Being of Parents Who Have Experienced Traumatic Childbirth: A Systematic Review and Meta-Analysis}

Shorey, Shefaly, Downe, Soo, Chua, Joelle Yan Xin, Byrne, Sofia O., Fobelets, Maaike and Lalor, Joan Gabrielle

Available at http://clok.uclan.ac.uk/40205/

Shorey, Shefaly, Downe, Soo ORCID: 0000-0003-2848-2550, Chua, Joelle Yan Xin, Byrne, Sofia O., Fobelets, Maaike and Lalor, Joan Gabrielle (2021)

Effectiveness of Psychological Interventions to Improve the Mental Well-Being of Parents Who Have Experienced Traumatic Childbirth: A Systematic Review and Meta-Analysis. Trauma, Violence and Abuse. ISSN 1524-8380

It is advisable to refer to the publisher's version if you intend to cite from the work. http://dx.doi.org/10.1177/15248380211060808

For more information about UCLan's research in this area go to http://www.uclan.ac.uk/researchgroups/ and search for <name of research Group>.

For information about Research generally at UCLan please go to http://www.uclan.ac.uk/research/

All outputs in CLoK are protected by Intellectual Property Rights law, including Copyright law. Copyright, IPR and Moral Rights for the works on this site are retained by the individual authors and/or other copyright owners. Terms and conditions for use of this material are defined in the policies page. 


\title{
Effectiveness of Psychological Interventions to Improve the Mental Well-Being of Parents Who Have Experienced Traumatic Childbirth: A Systematic Review and Meta- Analysis
}

\author{
Shefaly Shorey (D) ${ }^{1}$, Soo Downe (D) ${ }^{2}$, Joelle YanJoelle Yan Xin Xin Chutachua ${ }^{1}$, Sofia O. Byrne (D) ${ }^{3}$, Maaike \\ Fobelets ${ }^{4,5}$ and Joan Joan Gabrielle Lalor ${ }^{3}$
}

\author{
${ }^{1}$ Alice Lee Centre for Nursing Studies, Yong Loo Lin School of Medicine, National University of Singapore, Level \\ 2, Clinical Research Centre, Singapore \\ ${ }^{2}$ School of Community Health and Midwifery, University of Central Lancashire, Preston, UK \\ ${ }^{3}$ Trinity College Dublin, Dublin 2, Ireland \\ ${ }^{4}$ Faculty of Medicine and Pharmacy, Department of Public Health Sciences, Biostatistics and Medical Informatics \\ (BISI) Research Group, Vrije Universiteit Brussel, Brussels, Belgium \\ ${ }^{5}$ Department Health Care, Knowledge Centre Brussels Integrated Care, Erasmus University College Brussels, \\ Brussels, Belgium
}

Shefaly Shorey, PhD, RN, RM, Assistant Professor, Alice Lee Centre for Nursing Studies, Yong Loo Lin School of Medicine, National University of Singapore, Level 2, Clinical Research Centre, Block MD11 10 Medical Drive, 117597, Singapore. Email: nurssh@nus.edu.sg

\begin{abstract}
Considering the adverse impact that traumatic childbirth experiences can have on parental mental wellbeing, studies that have investigated the potential of providing postnatal psychological support for this group of parents require evaluation. This systematic review aimed to examine the effectiveness of psychological interventions at improving the mental well-being of parents who have experienced traumatic childbirth in terms of anxiety, depression, fear of childbirth, and post-traumatic stress disorder (PTSD) symptoms. Seven electronic databases were searched from their respective inception dates up to January 2021. Only quantitative studies that reported the effects of psychological interventions on anxiety, depression, fear of childbirth, and/or PTSD symptoms in selective (at risk of traumatic childbirth experience) or indicated (selfdefined childbirth experience as traumatic for any reason) populations of parents (mothers and/or fathers) were included. Eight studies were included and meta-analyses were conducted using a random-effect model. All studies were conducted on mothers only, and one study had minimal father involvement. Results showed that psychological interventions were more effective in reducing fear of childbirth and improving PTSD symptoms compared to anxiety and depression. Greater improvement in depression was reported at 3-8 weeks' follow-up than at immediate post-intervention. Subgroup analyses showed that technologybased interventions were feasible, and indicated interventions were more effective than selective interventions. Conducting future interventions in more geographical regions, engaging and including fathers more actively, incorporating both personalized professional therapy and informal peer support, striving for flexibility and convenience, as well as addressing topics on self-doubt and coping skills can improve current interventions.
\end{abstract}

\section{Keywords:}

psychological, mental well-being, parent, trauma, childbirth, post-traumatic stress disorder, meta-analysis 
Award Recipient :

\section{Summary of Critical Findings}

- Psychological interventions were more effective in reducing fear of childbirth and improving PTSD symptoms among mothers who have experienced traumatic childbirth as compared to anxiety and depression.

- Depression improved only around 3-8 weeks after the completion of psychological interventions and highlighted the need to support parents during this crucial period after traumatic childbirth.

- Technology-based interventions have successfully improved the mental well-being of mothers who experienced traumatic childbirth.

- Indicated interventions were more effective than selective interventions.

\section{Summary of Implications for Future Research and Practice}

- Future trials should be conducted in various geographical regions such as Asia, Africa, and America.

- Fathers need to be actively involved in future postnatal psychological interventions.

- Addressing issues of self-doubt and coping skills can help prevent depression and anxiety.

- A combination of group-based peer support as well as one-on-one personalized therapy may be more beneficial.

- Postnatal psychological support should prioritize flexibility and convenience.

\section{Introduction}

\section{Background}

Although most women perceive childbirth as a joyous occasion, some perceive their experience as negative. Studies have shown that about $30-43 \%$ of women perceived their childbirth experience as traumatic (Alcorn et al., 2010; DEVOTION CA18211, 2020) and 3.1-4\% of postnatal women go on to develop post-traumatic stress disorder (PTSD) (DEVOTION CA18211, 2020; Grekin \& O'Hara, 2014). The symptoms of postpartum PTSD include reexperiencing their birth experience in their own minds, avoiding medical settings that remind them of their traumatic birth experience, negative cognition and mood, and hyperarousal (Regier et al., 2013). Studies have found that operative birth methods (including emergency cesarean sections and instrumental vaginal deliveries via forceps or vacuum) (Eide et al., 2019; J. Söderquist et al., 2002), poor pain control (Grekin \& O'Hara, 2014), and maternal complications such as preeclampsia or postpartum hemorrhage (Furuta et al., 2012; Grekin \& O’Hara, 2014) contributed significantly to mothers' perception of their childbirth as traumatic. Interactions with healthcare professionals that are perceived as disrespectful, lacking in empathy for mothers and unsupportive of mothers' preferences for care during the perinatal period have also been associated with a negative/traumatic childbirth experience (Grekin \& O'Hara, 2014; Shorey \& Wong, 2020).

Women tend to develop more mental health complications such as postpartum anxiety (Leach et al., 2017), depression (Blom et al., 2010), fear of childbirth (Fairbrother et al., 2018), and PTSD (Grekin \& O'Hara, 2014) after experiencing a traumatic childbirth (Johan Söderquist et al., 2006), and these can incur high medical and social costs ( Bauer et al., 2014). Anxiety, depression, and PTSD have been established as multi-morbidities occurring among postnatal women (Agius et al., 2016; Dikmen-Yildiz et al., 2017). Moreover, anxiety and depression have been positively associated with fear of childbirth (tokophobia) (Storksen et al., 2012), and fear of childbirth, in particular, has been established as a powerful risk factor for the development of postpartum PTSD (Dikmen-Yildiz et al., 2018). In light of the close links among these mental health disorders, support provided to mothers after a traumatic childbirth should seek to address all these interrelated issues.

After a traumatic childbirth experience, mothers may experience nightmares and flashbacks, bouts of rage, irritability, concentration difficulties, and disrupted sleep, resulting in sleep deprivation, reduced libido and they may begin to participate less in the care of their child/children (Beck, 2016; Blasio et al., 2009). In the long term, poor maternal mental health can negatively affect their child's physical, cognitive, and social development (D'Souza et al., 2019; Giallo et al., 2015; Glasheen et al., 2010). Fathers who witnessed their partners' suffering might also experience secondhand psychological distress (Inglis et al., 2016). Moreover, the lack of sexual intercourse and difficulties in 
parenting have been reported to strain the relationship of many couples and end some marriages (Cava-Tadik et al., 2020; Shorey et al., 2019). In some cases of severe tokophobia, women suppressed their desire to have more children, which can also cause tension in the marital relationship (Shorey et al., 2018). As traumatic childbirth experience affects mothers and fathers, the mental well-being of both parents needs to be addressed.

Researchers and healthcare professionals have adopted both selective and indicated psychological interventions to help improve the mental well-being of parents who are susceptible to develop postpartum PTSD. Selective interventions are conducted for parents at risk of traumatic childbirth (Sheen \& Slade, 2015) after undergoing operative birth procedures (emergency cesarean sections or instrumental vaginal deliveries using forceps or vacuum) (Eide et al., 2019; J. Söderquist et al., 2002), and/or labor-related complications (poor pain control, preeclampsia or postpartum hemorrhage) (Furuta et al., 2012; Grekin \& O'Hara, 2014). On the other hand, indicated interventions are conducted for parents who self-defined their childbirth experience as traumatic for any reason (Sheen \& Slade, 2015). A psychological intervention refers to any psychotherapeutic method aimed at changing one's cognition, perception, and/or behavior (Eccleston et al., 2015). Some methods that have been conducted with these parents include debriefing, counseling, cognitive behavioral therapy (CBT)-based program, mindfulness-based program, and engagement of visuospatial cognitive tasks (Asadzadeh et al., 2020; Ayers et al., 2006; Bavelier \& Green, 2003; Holmes et al., 2009; Horsch et al., 2017; Meades et al., 2011; Pour-Edalati et al., 2018). They can be conducted either face-to-face in a group or one-on-one at the patient's home, healthcare institution, or online. Psychological interventions have been shown to help parents accept their negative childbirth experience, process their emotions and thoughts, and reframe their rumination on negative thoughts to focus on more productive and positive ones (Baer, 2003; Bryant, 2007; Meades et al., 2011). Hence, they can help parents improve multiple aspects of their mental health (anxiety, depression, fear of childbirth, and PTSD symptoms).

\section{Current Literature}

There is a lack of systematic reviews which focus on investigating the effectiveness of psychological interventions to improve the mental well-being of parents who have experienced traumatic childbirth. The review by Borg Cunen et al. (2014) only examined the effect of midwife-led interventions, while other reviews limited their focus to specific types of psychological interventions such as debriefing (Bastos et al., 2015), both debriefing and counseling (Gamble et al., 2002; Rowan et al., 2007; Sheen \& Slade, 2015) and expressive writing (Qian et al., 2020). Although six previous reviews examined the effectiveness of all types of psychological interventions on maternal postnatal mental health (de Bruijn et al., 2020; de Graaff et al., 2018; Furuta et al., 2018; Lapp et al., 2010; Peeler et al., 2013; Slade, Molyneux, et al., 2020), only two reviews focused on mothers who experienced a traumatic childbirth (de Bruijn et al., 2020; Slade, Molyneux, et al., 2020). Neither of these reviews included fathers, and in both reviews, PTSD was the only outcome of interest (de Bruijn et al., 2020; Slade, Molyneux, et al., 2020). Hence, the effect of psychological interventions on the other important and interrelated postnatal mental health outcomes such as anxiety, depression, and fear of childbirth in both mothers and fathers who experienced traumatic childbirth have not been examined. Therefore, this meta-analysis seeks to evaluate the overall effect of psychological interventions to improve anxiety, depression, fear of childbirth, and PTSD symptoms in parents (mothers and fathers) who experienced traumatic childbirth (Egger \& Smith, 1997).

\section{Aim}

This review aimed to determine the effectiveness of psychological interventions to reduce anxiety, depression, fear of childbirth, and PTSD symptoms at immediate post-intervention (primary outcomes) and any follow-up timepoint (secondary outcomes) in both mothers and fathers who have experienced traumatic childbirth.

\section{Method}

This review followed the Preferred Reporting Items for Systematic Reviews and Meta-Analyses (PRISMA) guidelines (Moher et al., 2009), and the PRISMA checklist can be found in Online Appendix A. A protocol can be found on the PROSPERO website (CRD42021230450).

\section{Eligibility Criteria}

Population Selective and indicated populations of parents (mother and/or father) who delivered liveborn infant or infants (including twins) following a traumatic childbirth index pregnancy were included. This refers to parents who are at risk of perceiving their childbirth experience as traumatic following any of the aforementioned operative birth procedure and/or labor-related complications (selective), and parents who have self-defined their birth experience as traumatic (indicated). Parents who developed anxiety, depressive symptoms, fear of childbirth, PTSD symptoms, or other signs of psychological conditions only after a traumatic childbirth were also included. Conversely, parents with pre-existing mental illnesses and/or history of substance abuse before childbirth (before and/or during pregnancy), and those who experienced a stillbirth or miscarriage in the index pregnancy were excluded. In addition, parents with multiple births who suffered the loss of at least one child were excluded. Studies that exclusively recruited parents who had traumatic experiences specifically related to preterm babies, such as experience in the neonatal intensive care unit, 
were excluded in order to focus on parents whose psychological distress was due to the traumatic birth experience

rather than the subsequent poor infant outcomes.

Intervention Any selective or indicated psychological intervention delivered to either mother and/or father with the aim to improve their mental well-being at any timepoint after their traumatic childbirth experience, but before their next pregnancy.

Comparator Studies with control groups undergoing the standard postpartum care offered by their respective healthcare facility, or wait-list control were included. Any non-psychological or psychological intervention with an aim to improve parents' mental well-being was excluded.

Outcomes Included studies must measure any or all of the following: anxiety, depression, fear of childbirth or PTSD symptoms at pre- and post-intervention. Outcomes could be assessed by any method of measurement such as sweat test, skin conduction test, or self-reported by participants, and had to be reported in terms of mean and standard deviation (SD) or other forms that can be transformed into mean (SD) or where raw study data could be obtained from study authors. The immediate post-intervention values of the four outcomes were of primary focus, while follow-up measurements at other timepoints were viewed as secondary interests.

Study Design Only randomized controlled trials (RCTs), cluster RCTs and controlled clinical trials (CCTs) were included.

Language and Publication Status Only peer-reviewed journal articles and unpublished dissertations written in any language were included. Papers that conducted secondary re-analysis of data specific to review outcomes were also included.

\section{Study Selection}

The following electronic databases were searched from their respective date of inception to January 2021: PubMed, Embase, CINAHL, PsycINFO, Scopus, Web of Science, and ProQuest Dissertations \& Theses Global. The reference lists of similar reviews and included studies were examined, and backward searching was done to identify other relevant trials. Upon encountering potentially relevant studies with unavailable full-text, request for a full-text was sent to the respective authors. Endnote Version X8 was used to organize the records according to their databases and remove duplicates. The eligibility criteria were used to screen all titles and abstracts of the records retrieved from the search. Potentially relevant studies were identified and had their full-texts scrutinized to determine their relevancy. Google translate was used to translate foreign language texts into English. Two independent reviewers (SS and CJYX) conducted this process and judgment discrepancies were resolved via discussions. A sample search strategy for Embase database is shown in Online Appendix B.

\section{Data Extraction}

A data extraction form was used to extract key details of included studies such as sample size, study design, outcomes measured, intervention content, duration and method of delivery, and attrition rate. The mean and SD values of the review's outcomes were also extracted and relevant formulas were used to transform the data to obtain these specific values when necessary (Higgins \& Green, 2011). Data transformation of the mean and interquartile values in one of the included studies (Ryding et al., 1998) was performed by using other relevant formulas (Luo et al., 2018; Shi et al., 2020 ). Discussions were held to resolve any disagreements between the two independent reviewers (SS and CJYX).

\section{Quality Appraisal}

The Cochrane Risk of Bias tool was used to assess five types of biases for all included studies: selection bias, performance bias, detection bias, attrition bias, and reporting bias (Higgins \& Green, 2011). The overall bias rating for each study was decided by the worst score it received for any domain (Kempler et al., 2016). Publication bias was not examined as no forest plot contained at least 10 trials to produce a meaningful funnel plot (Sterne et al., 2011).

The Grades of Recommendation, Assessment, Development, and Evaluation (GRADE) approach assessed the quality of evidence at the outcome level. The quality rating depended on five factors: risk of bias of individual studies, inconsistency, directness of evidence, precision of effect estimates, and publication bias (Higgins \& Green, 2011). The online GRADEpro software rated each outcome separately (GRADEpro, 2015). Quality appraisal was conducted by two independent reviewers (SS and CJYX) and disagreements were discussed until consensus was met.

\section{Data Synthesis}

Characteristics of the included studies and interventions were summarized narratively. Data of the same outcomes were pooled using meta-analyses under the random-effect model. Since the included studies used different scales to report outcomes using continuous data, the Review Manager software 5.4 was used to calculate the standardized mean differences (SMD) and 95\% confidence intervals (CI) under the inverse-variance method (Higgins \& Green, 2011). 
Effect sizes were interpreted as very small (0.1), small (0.2), medium (0.5), large (0.8), very large (1.2), and huge (2.0) (Sawilowsky, 2009). As one study used two scales to measure PTSD symptoms in mothers, only the Impact of Event Scale-Reversed scores were included in this review as the scale assesses a more general measure of PTSD symptoms and is similar to the Acute Stress Disorder Scale, Impact of Event Scale and Post-traumatic Stress Disorder Checklist-5, which were used by the other studies; on the other hand, the Traumatic Event Scale is different and was excluded ( Nieminen et al., 2016). As significant baseline differences between the intervention and control groups were reported for the fear of childbirth in Pour-Edalati et al., (2018), and depression and PTSD symptoms in Meades et al., (2011), these two studies were excluded from the meta-analyses and had their results synthesized narratively.

Heterogeneity of results were assessed using the $\mathrm{I}^{2}$ statistic and Cochran $\mathrm{Q}$ chi-squared test. $\mathrm{I}^{2}$ values were categorized as low importance $(\leq 40 \%)$, moderate $(30-60 \%)$, substantial $(50-90 \%)$, and considerable $(75-100 \%)$. Statistically significant heterogeneity of the chi-squared test was identified when its corresponding p-value was less than 0.10 (Higgins \& Green, 2011). Abdollahpour et al. (2018) compared two different interventions (one CBT and one debriefing) with one control group hence the number of participants in the control group was randomly split during meta-analysis to avoid unit-of-analysis error (Higgins \& Green, 2011). Subgroup analyses were conducted to reduce heterogeneity levels and examine the effect certain variables have on the review outcomes (Higgins \& Green, 2011; Sedgwick, 2013). The variables examined were technology-based intervention versus non-technology-based intervention, psychological intervention type, and selective intervention versus indicated intervention. As the prespecified subgroup analyses variables listed in the protocol submitted to PROSPERO were changed during the course of this study, the current subgroup analyses were hence conducted with the purpose of generating hypotheses.

\section{Results}

\section{Search Outcomes}

The search from the seven listed electronic databases, and relevant reviews and articles retrieved 7865 articles. After the deletion of 3739 duplicates and the exclusion of 4060 articles based on their titles and abstracts, 66 articles were left for full-text screening. Examination of full-texts excluded another 58 articles due to reasons such as lack of a control group or interventions were conducted on parents who did not experience traumatic childbirth hence there were eight peerreviewed primary studies included in this review (Abdollahpour et al., 2018; Asadzadeh et al., 2020; Horsch et al., 2017; Kershaw et al., 2005; Meades et al., 2011; Nieminen et al., 2016; Pour-Edalati et al., 2018; Ryding et al., 1998). As one study reported two trials that conducted two different interventions (one CBT and one debriefing) ( Abdollahpour et al., 2018), the eight studies reported nine trials in total. The search outcomes and reasons for exclusion of studies are shown as a PRISMA flow diagram in Figure 1.

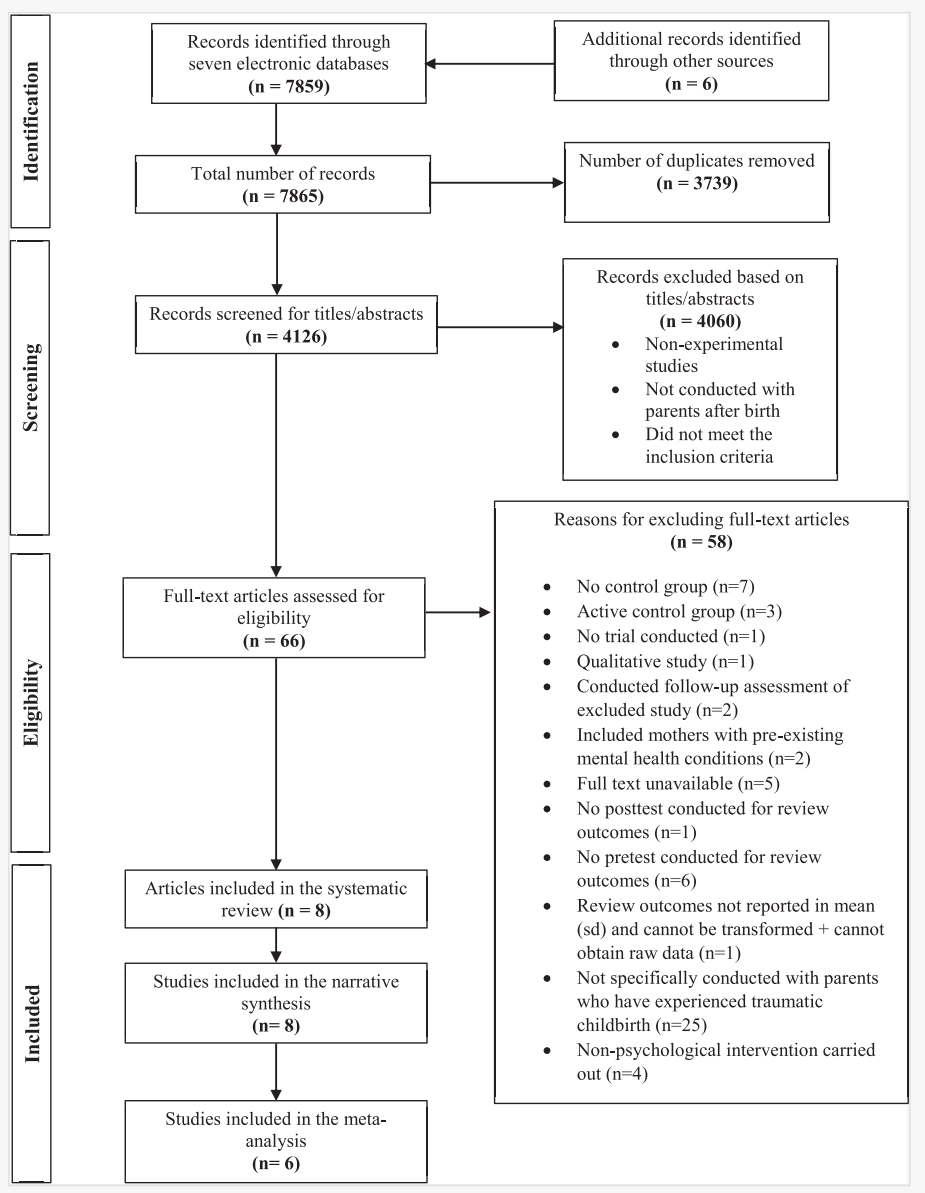




\section{Characteristics of the Included Studies}

Out of the eight included studies, there were seven RCTs and one CCT (Meades et al., 2011). They were conducted in four different countries: Iran $(n=3)$, Switzerland $(n=1)$, Sweden $(n=2)$, and England $(n=2)$. Three included studies conducted selective interventions as they recruited women at risk for traumatic childbirths after undergoing operative birth methods (Kershaw et al., 2005) or emergency caesarean sections (Horsch et al., 2017; Ryding et al., 1998), while the rest conducted indicated interventions by targeting women pre-screened for self-defined traumatic childbirths (Abdollahpour et al., 2018; Asadzadeh et al., 2020; Meades et al., 2011; Nieminen et al., 2016) or significant fear of childbirth caused by previous traumatic birth experience (Pour-Edalati et al., 2018). The types of psychological interventions included debriefing (Abdollahpour et al., 2018; Kershaw et al., 2005; Meades et al., 2011), counseling (Asadzadeh et al., 2020; Ryding et al., 1998), CBT-based (Abdollahpour et al., 2018; Nieminen et al., 2016 ), mindfulness-based (Pour-Edalati et al., 2018), and a visuospatial cognitive task (“Tetris” game) to reduce participants' intrusive traumatic memories (Horsch et al., 2017). Only Pour-Edalati et al. (2018) conducted a group-based intervention while the other studies conducted one-on-one interventions. Seven studies conducted interventions inperson; one had an additional telephone counseling session (Asadzadeh et al., 2020). The last study developed an online intervention with homework assignments for participants to complete independently at home (Nieminen et al., 2016). All studies only engaged mothers except for Ryding et al. (1998) which allowed fathers to ask questions after the last counseling session. The summarized characteristics and more detailed characteristics of the included studies can be found in Table 1 and Online Appendix C, respectively.

\section{Table 1.}

(i) The table layout displayed in this section is not how it will appear in the final version. The representation below is solely purposed for providing corrections to the table. To preview the actual presentation of the table, please view the Proof.

Summary of included studies.

\begin{tabular}{|c|c|c|c|c|}
\hline $\begin{array}{l}\text { Study } \\
\text { (Country) }\end{array}$ & $\begin{array}{l}\text { Research } \\
\text { Design/Arms }\end{array}$ & Participants Number and Type of Traumatic Birth & $\begin{array}{l}\text { Intervention } \\
\text { Duration (Follow- } \\
\text { Up)/Father } \\
\text { Involvement }\end{array}$ & $\begin{array}{l}\text { Review } \\
\text { outcomes: } \\
\text { 1.Anxiety } \\
\text { 2.Depression } \\
\text { 3.Fear of } \\
\text { childbirth } \\
\text { 4.PTSD } \\
\text { Symptoms }\end{array}$ \\
\hline $\begin{array}{l}\text { Abdollahpour } \\
\text { et al., } 2018 \\
\text { (Iran) }\end{array}$ & $\begin{array}{l}3 \text { arm RCT } \\
\text { 1.Debriefing } \\
\text { 2.Brief CBT } \\
\text { 3.Standard } \\
\text { care }\end{array}$ & $\begin{array}{l}193 \text { women screened for traumatic birth (met criterion A } \\
\text { of DSM-5 for PTSD) }\end{array}$ & $\begin{array}{l}1 \text { session lasting } 40- \\
60 \text { min within } 48 \mathrm{hrs} \\
\text { postpartum } \\
\text { (1-1.5 months) } \\
\text { No father } \\
\text { involvement }\end{array}$ & $\begin{array}{l}\text { 1.No } \\
\text { 2.Yes } \\
\text { 3.No } \\
\text { 4.No }\end{array}$ \\
\hline $\begin{array}{l}\text { Asadzadeh et } \\
\text { al., } 2020 \\
\text { (Iran) }\end{array}$ & $\begin{array}{l}2 \text { arm RCT } \\
\text { 1.Counseling } \\
\text { 2.Standard } \\
\text { care }\end{array}$ & $\begin{array}{l}90 \text { women screened for traumatic birth (met criterion A of } \\
\text { DSM- } 5 \text { for PTSD) }\end{array}$ & $\begin{array}{l}1 \text { session within } \\
72 \mathrm{hrs} \text { after birth }+ \\
\text { Telephone session } \\
\text { 4-6 weeks } \\
\text { postpartum } \\
\text { Each session lasts } \\
40-60 \text { min } \\
\text { (1-1.5 months) } \\
\text { No father } \\
\text { involvement }\end{array}$ & $\begin{array}{l}\text { 1.Yes } \\
\text { 2.Yes } \\
\text { 3.No } \\
\text { 4.Yes }\end{array}$ \\
\hline $\begin{array}{l}\text { Horsch et al., } \\
2017 \\
\text { (Switzerland) }\end{array}$ & $\begin{array}{l}2 \text { arm RCT } \\
\text { 1.Visuospatial } \\
\text { cognitive task } \\
\text { (Tetris game) } \\
\text { 2.Standard } \\
\text { care }\end{array}$ & $\begin{array}{l}56 \text { women who have just given birth by emergency } \\
\text { caesarean (did not use criterion A of DSM- } 5 \text { for PTSD to } \\
\text { screen for participants) }\end{array}$ & $\begin{array}{l}1 \text { session (at least } \\
10 \mathrm{~min} \text { ) within } 6 \mathrm{hrs} \\
\text { postpartum } \\
\text { (3 weeks) } \\
\text { No father } \\
\text { involvement }\end{array}$ & $\begin{array}{l}\text { 1.Yes } \\
\text { 2.Yes } \\
\text { 3.No } \\
\text { 4.Yes }\end{array}$ \\
\hline $\begin{array}{l}\text { Kershaw et } \\
\text { al., } 2005 \\
\text { (England) }\end{array}$ & $\begin{array}{l}2 \text { arm RCT } \\
\text { 1.Community } \\
\text { debriefing } \\
2 . \text { Standard } \\
\text { care }\end{array}$ & $\begin{array}{l}319 \text { women who delivered first child by operative birth } \\
\text { methods (forceps, vacuum, emergency caesarean) } \\
\text { (did not use criterion A of DSM- } 5 \text { for PTSD to screen for } \\
\text { participants) }\end{array}$ & $\begin{array}{l}2 \text { sessions } 10 \text { weeks } \\
\text { apart } \\
\text { (10 weeks) } \\
\text { No father } \\
\text { involvement }\end{array}$ & $\begin{array}{l}\text { 1.No } \\
\text { 2.No } \\
\text { 3.Yes } \\
\text { 4.Yes }\end{array}$ \\
\hline Meades et al., & 2 arm CCT & 80 women screened for traumatic birth (met criterion A & $1-1.5 \mathrm{hr}$ session & 1.No \\
\hline
\end{tabular}




\begin{tabular}{|c|c|c|c|c|}
\hline $\begin{array}{l}2011 \\
\text { (England) }\end{array}$ & $\begin{array}{l}\text { 1.Postnatal } \\
\text { debrief } \\
\text { 2.Standard } \\
\text { care }\end{array}$ & for PTSD) & $\begin{array}{l}\text { (No follow-up) } \\
\text { No father } \\
\text { involvement }\end{array}$ & $\begin{array}{l}\text { 2.Yes } \\
\text { 3.No } \\
\text { 4.Yes }\end{array}$ \\
\hline $\begin{array}{l}\text { Nieminen et } \\
\text { al., } 2016 \\
\text { (Sweden) }\end{array}$ & $\begin{array}{l}2 \text { arm RCT } \\
\text { 1.Trauma- } \\
\text { focused ICBT } \\
\text { program } \\
\text { 2.Wait-list } \\
\text { control }\end{array}$ & $\begin{array}{l}56 \text { women screened for traumatic birth }(\mathrm{TES} \geq 30 \text { ) } \\
\text { (did not use criterion A of DSM- } 5 \text { for PTSD to screen for } \\
\text { participants) }\end{array}$ & $\begin{array}{l}8 \text { weekly modules } \\
\text { (No follow-up) } \\
\text { No father } \\
\text { involvement }\end{array}$ & $\begin{array}{l}\text { 1.Yes } \\
\text { 2.Yes } \\
\text { 3.No } \\
\text { 4.Yes }\end{array}$ \\
\hline $\begin{array}{l}\text { Pour-Edalati } \\
\text { et al., } 2018 \\
\text { (Iran) }\end{array}$ & $\begin{array}{l}2 \text { arm RCT } \\
\text { 1.MBSR } \\
\text { training } \\
\text { 2.Control }\end{array}$ & $\begin{array}{l}48 \text { single-child women with history of normal birth } \\
\text { methods who had not become pregnant again after at } \\
\text { least } 6 \text { years due to fear of childbirth (did not use } \\
\text { criterion A of DSM- } 5 \text { for PTSD to screen for participants) }\end{array}$ & $\begin{array}{l}8 \times 90 \mathrm{~min} \text { twice a } \\
\text { week } \\
\text { (No follow-up) } \\
\text { No father } \\
\text { involvement }\end{array}$ & $\begin{array}{l}\text { 1.No } \\
\text { 2.No } \\
\text { 3.Yes } \\
\text { 4.No }\end{array}$ \\
\hline $\begin{array}{l}\text { Ryding et al., } \\
1998 \\
\text { (Sweden) }\end{array}$ & $\begin{array}{l}2 \text { arm RCT } \\
\text { 1.Counseling } \\
\text { 2.Standard } \\
\text { care }\end{array}$ & $\begin{array}{l}105 \text { women who have just given birth by emergency } \\
\text { caesarean (did not use criterion A of DSM- } 5 \text { for PTSD to } \\
\text { screen for participants) }\end{array}$ & $\begin{array}{l}1 \mathrm{hr} \text { session }+ \\
3 \mathrm{X} 45 \text { min sessions } \\
\text { in } 3 \text { weeks } \\
\text { ( } 5 \text { months) } \\
\text { Father invited to ask } \\
\text { questions after last } \\
\text { session }\end{array}$ & $\begin{array}{l}\text { 1.No } \\
\text { 2.No } \\
\text { 3.Yes } \\
\text { 4.Yes }\end{array}$ \\
\hline
\end{tabular}

Note. CBT: Cognitive behavioral therapy; CCT: Controlled Clinical Trial; DSM: Diagnostic and Statistical Manual; ICBT: Internetbased cognitive behavioral therapy; PTSD: Post-traumatic Stress Disorder; RCT: Randomized Controlled Trial; TES: Traumatic Event Scale.

\section{Quality Appraisal}

The Cochrane risk of bias tool was used to assess all included studies. Two studies were rated as low overall bias ( Abdollahpour et al., 2018; Asadzadeh et al., 2020), one study was rated as having unclear overall bias (Nieminen et al., 2016), and the remaining studies had high overall bias. The two independent reviewers had an inter-rater agreement of approximately $97 \%$ and a Cohen's kappa value of 0.95 . The risk of bias summary is presented in Figure 2. Quality appraisal at the outcome level, conducted using the GRADE approach, rated the outcomes of anxiety, depression, and PTSD symptoms as very low quality while the fear of childbirth outcome was rated as low quality. More details about the GRADE ratings are presented in Online Appendix D. 


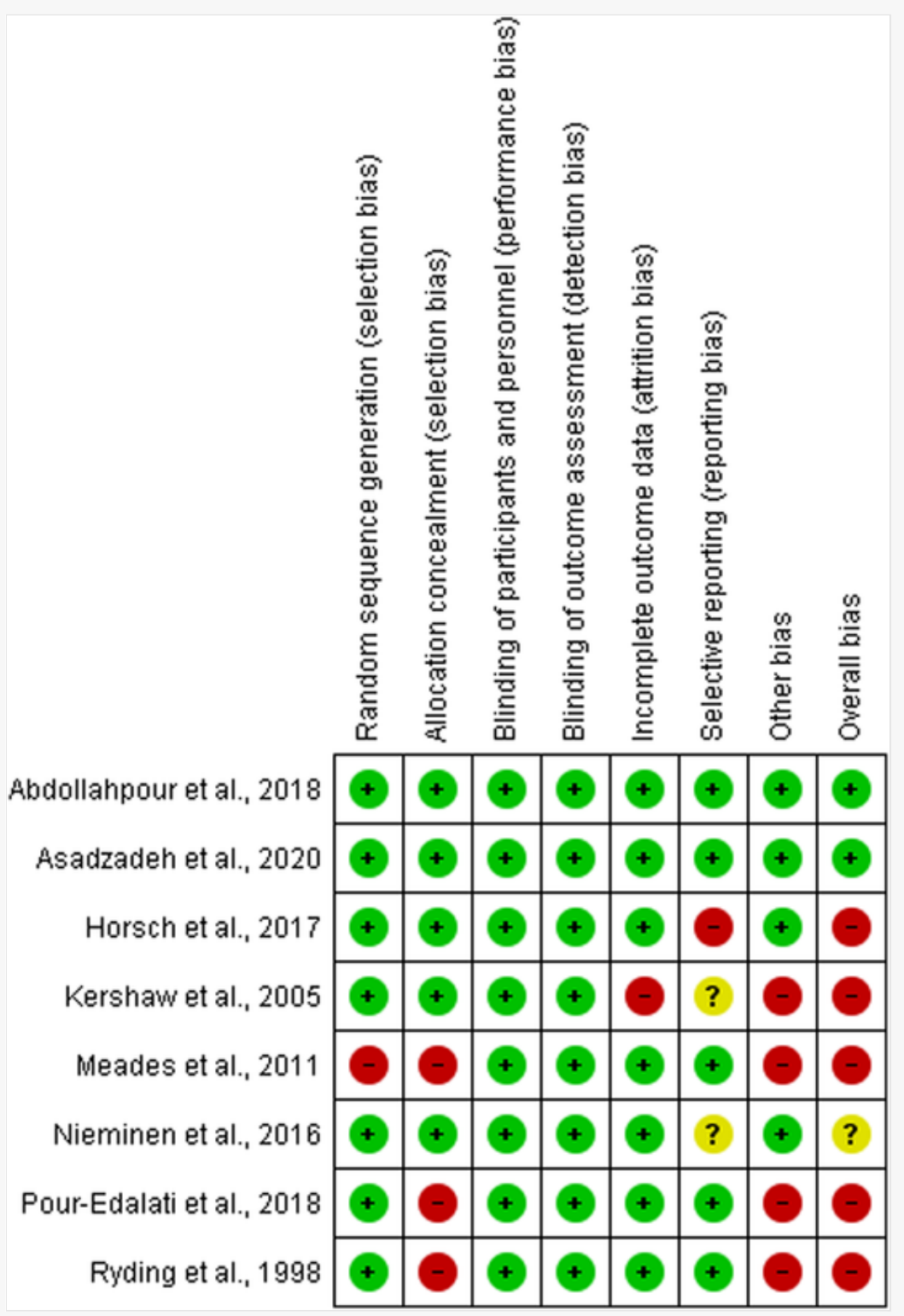

Risk of bias summary.

\section{Anxiety}

The meta-analysis conducted for the three studies that assessed anxiety at immediate post-intervention (Asadzadeh et al., 2020; Horsch et al., 2017; Nieminen et al., 2016) showed a statistically non-significant medium effect favoring the intervention group (SMD $=-0.54,95 \% \mathrm{CI}:-1.17$ to $0.09, \mathrm{Z}=1.68, p=0.09$ ) (Figure 3 ).

\section{Figure 3.}

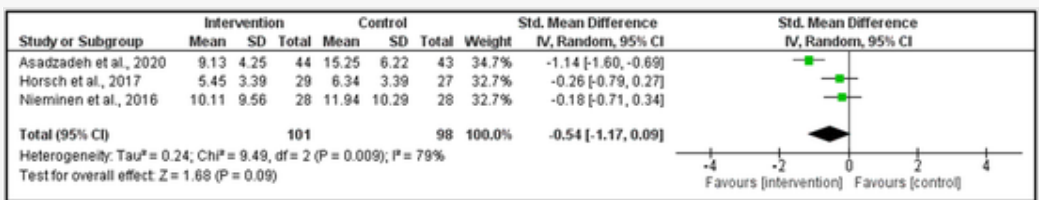

Forest plot of anxiety at post-intervention in three included studies.

The subgroup analysis of technology-based intervention versus non-technology-based intervention showed statistically significant substantial subgroup differences $\left(\mathrm{I}^{2}=89.4 \%, p=0.002\right)$. The technology-based subgroup reported a statistically non-significant small effect with low heterogeneity $\left(\mathrm{I}^{2}=0 \%, p=0.84\right)$ while the nontechnology-based subgroup consisting of one study reported a statistically significant large effect (Online Appendix E.1).

The subgroup analysis of psychological intervention type showed statistically significant substantial subgroup differences ( $\left.\mathrm{I}^{2}=78.9 \%, p=0.009\right)$. The debriefing/counseling, CBT and visuospatial cognitive task subgroups only had one study each that reported a statistically significant large effect, a statistically non-significant very small effect and a statistically non-significant small effect, respectively (Online Appendix E.2). 
The subgroup analysis of selective intervention versus indicated intervention showed statistically nonsignificant subgroup differences $\left(\mathrm{I}^{2}=0 \%, p=0.45\right)$. The selective intervention subgroup reported a statistically nonsignificant small effect while the indicated intervention subgroup reported a statistically non-significant medium effect (Online Appendix E.3).

The meta-analysis conducted for the two studies that assessed anxiety at follow-up duration ranging from 3 to 8 weeks (Asadzadeh et al., 2020; Horsch et al., 2017) showed a statistically non-significant medium effect favoring the intervention group ( $\mathrm{SMD}=-0.59,95 \% \mathrm{CI}:-1.45$ to $0.27, \mathrm{Z}=1.35, p=0.18$ ), with substantial statistical heterogeneity $\left(\mathrm{I}^{2}=84 \%, p=0.01\right)$ ( Online Appendix F).

\section{Depression}

The meta-analysis conducted for the four studies reporting on five trials which assessed depression at immediate postintervention (Abdollahpour et al., 2018; Asadzadeh et al., 2020; Horsch et al., 2017; Nieminen et al., 2016) showed a statistically non-significant very small effect favoring the intervention group (SMD $=-0.18,95 \% \mathrm{CI}:-0.80$ to $0.44, \mathrm{Z}$ $=0.56, p=0.57)$, with substantial statistical heterogeneity $\left(\mathrm{I}^{2}=89 \%, p<0.00001\right)$ (Figure 4).

Figure 4.

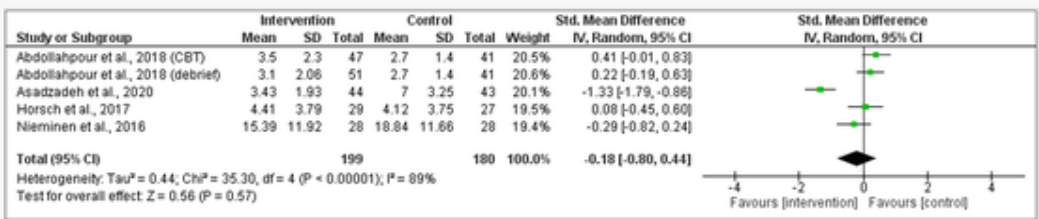

Forest plot of depression at post-intervention in four included studies reporting five trials in total.

The subgroup analysis of technology-based intervention versus non-technology-based intervention showed statistically non-significant subgroup differences $\left(\mathrm{I}^{2}=0 \%, p=0.83\right)$. The technology-based subgroup reported a statistically non-significant very small effect while the non-technology-based subgroup reported a statistically nonsignificant small effect (Online Appendix G.1).

The subgroup analysis of psychological intervention type showed statistically non-significant subgroup differences $\left(\mathrm{I}^{2}=0 \%, p=0.74\right)$. The debriefing/counseling subgroup reported a statistically non-significant medium effect while both the CBT and visuospatial cognitive task subgroups reported statistically non-significant negligible effect favoring the control group (Online Appendix G.2).

The subgroup analysis of selective intervention versus indicated intervention showed statistically nonsignificant subgroup differences $\left(\mathrm{I}^{2}=0 \%, p=0.50\right.$ ). The selective intervention subgroup reported a statistically nonsignificant negligible effect favoring the control group while the indicated intervention subgroup reported a statistically non-significant small effect (Online Appendix G.3 $)$.

The meta-analysis conducted for the three studies reporting on four trials which assessed depression at followup between 3 and 8 weeks (Abdollahpour et al., 2018; Asadzadeh et al., 2020; Horsch et al., 2017), showed a statistically non-significant medium effect favoring the intervention group ( $\mathrm{SMD}=-0.73,95 \% \mathrm{CI}:-1.51$ to $0.05, \mathrm{Z}=$ $1.83, p=0.07)$, with considerable statistical heterogeneity $\left(\mathrm{I}^{2}=91 \%, p<0.00001\right)$ ( $\left.\underline{\text { Online Appendix } \mathrm{H}}\right)$.

\section{Fear of Childbirth}

The meta-analysis conducted for the two studies that assessed fear of childbirth at immediate post-intervention ( Kershaw et al., 2005; Ryding et al., 1998) showed a statistically significant small effect favoring the intervention group $(\mathrm{SMD}=-0.37,95 \% \mathrm{CI}:-0.67$ to $-0.08, \mathrm{Z}=2.46, p=0.01)$, with low statistical heterogeneity $\left(\mathrm{I}^{2}=36 \%, p=0.21\right)($ Figure 5). Subgroup analysis was not performed as both studies conducted non-technology-based, debriefing/counseling and selective interventions. 


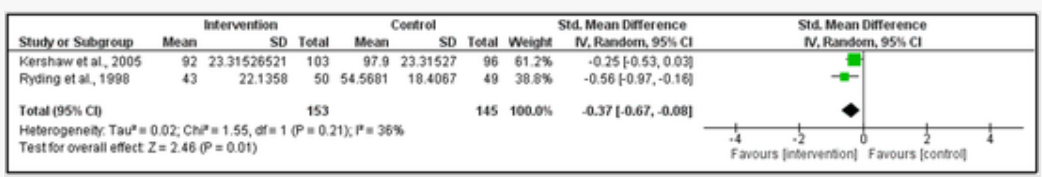

Forest plot of fear of childbirth at post-intervention in two included studies.

The meta-analysis conducted for the two studies that assessed fear of childbirth at follow-up between 2.5 and 5 months (Kershaw et al., 2005; Ryding et al., 1998) showed a statistically significant small effect favoring the intervention group (SMD $=-0.38,95 \% \mathrm{CI}:-0.61$ to $-0.15, \mathrm{Z}=3.23, p=0.001$ ), with low statistical heterogeneity $\left(\mathrm{I}^{2}=0 \%, p=0.40\right)(\underline{\text { Online Appendix I })}$.

\section{PTSD Symptoms}

The meta-analysis conducted for the five studies that assessed PTSD symptoms at immediate post-intervention ( Asadzadeh et al., 2020; Horsch et al., 2017; Kershaw et al., 2005; Nieminen et al., 2016; Ryding et al., 1998) showed a statistically significant medium effect favoring the intervention group ( $\mathrm{SMD}=-0.62,95 \% \mathrm{CI}:-0.98$ to $-0.26, \mathrm{Z}=$ $3.37, p=0.0008)$, with substantial statistical heterogeneity $\left(\mathrm{I}^{2}=72 \%, p=0.007\right)$ (Figure 6).

\section{Figure 6.}

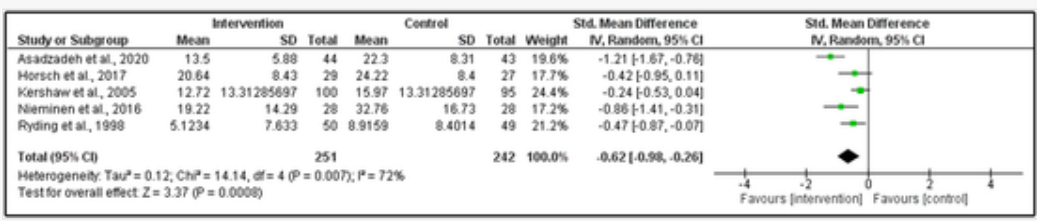

Forest plot of PTSD symptoms at post-intervention in five included studies.

The subgroup analysis of technology-based intervention versus non-technology-based intervention showed statistically non-significant subgroup differences $\left(\mathrm{I}^{2}=0 \%, p=0.97\right)$. Both the technology-based and the nontechnology-based subgroups reported a statistically significant medium effect (Online Appendix J.1).

The subgroup analysis of psychological intervention type showed statistically non-significant subgroup differences $\left(\mathrm{I}^{2}=0 \%, p=0.53\right)$. The debriefing/counseling subgroup reported a statistically significant medium effect while the CBT subgroup reported a statistically significant large effect; the visuospatial cognitive task subgroup reported a statistically non-significant small effect (Online Appendix J.2).

The subgroup analysis of selective intervention versus indicated intervention showed statistically significant considerable subgroup differences $\left(\mathrm{I}^{2}=91.8 \%, p=0.0005\right)$. The selective intervention subgroup reported a statistically significant small effect with low heterogeneity $\left(\mathrm{I}^{2}=0 \%, p=0.63\right)$ while the indicated intervention subgroup reported a statistically significant large effect with low heterogeneity $\left(\mathrm{I}^{2}=0 \%, p=0.33\right.$ ) (Online Appendix $\underline{\mathrm{J} .3}$ ).

Subgroup analysis of follow-up duration conducted for the four studies that assessed PTSD symptoms at various follow-up timepoints (Asadzadeh et al., 2020; Horsch et al., 2017; Kershaw et al., 2005; Ryding et al., 1998), showed statistically non-significant subgroup differences $\left(\mathrm{I}^{2}=0 \%, p=0.48\right)$. The "up to two months" subgroup reported a statistically non-significant medium effect, while the "more than two months" subgroup reported a statistically non-significant small effect (Online Appendix K).

\section{Narrative Synthesis}

Fear of childbirth assessed by Pour-Edalati et al. (2018) at pre-intervention showed significant differences between the intervention and control groups ( $\mathrm{p}$-value $<0.05$ ) and was hence excluded from meta-analysis. The mindfulness-based intervention group reported a statistically significant decrease in fear of childbirth scores at immediate post-intervention compared to baseline, whereas the control group did not (Pour-Edalati et al., 2018). No follow-up assessments were conducted.

Depression and PTSD symptoms assessed by Meades et al., (2011) at pre-intervention showed significant differences between the intervention and control groups ( $\mathrm{p}$-value $<0.05)$ and were hence excluded from meta-analysis. The debriefing group reported a statistically significant decrease in PTSD symptoms at immediate post-intervention compared to baseline, whereas the control group receiving standard care did not. Conversely, mothers in both groups reported similar reduction in depression scores at immediate post-intervention when compared to baseline (Meades et al., 2011). No follow-up assessments were conducted. 


\section{Discussion}

This review examined the effectiveness of psychological interventions aimed at improving the mental well-being (anxiety, depression, fear of childbirth, and PTSD symptoms) of parents who have experienced traumatic childbirth. However, as none of the studies actively engaged and included fathers, this review's findings can only be applied to mothers. Although mothers are more prone to psychological distress after a traumatic childbirth experience, a recent study reported that $7.2 \%$ of fathers showed signs of probable PTSD at 1-month postpartum, highlighting the need for postnatal psychological interventions to target both mothers and fathers (Horsch et al., 2020). Moreover, fathers have expressed their desire to receive postnatal psychological support (Inglis et al., 2016; Poh et al., 2014; Shorey \& Wong, 2020) and future trials should make an effort to do so. Furthermore, as included studies only represented mothers from Europe and the Middle East, future trials conducted in more diverse geographical regions would be needed to have a global understanding of parents who have experienced traumatic childbirth.

Immediate post-intervention results from meta-analyses showed that psychological interventions were more effective in decreasing PTSD symptoms and fear of childbirth among women who have experienced traumatic childbirth since statistically significant medium and small effects were observed, respectively, compared to anxiety and depression which showed statistically non-significant medium and very small effects, respectively. The two studies that were narratively summarized also reported a reduction in fear of childbirth (Pour-Edalati et al., 2018) and an improvement in PTSD symptoms (Meades et al., 2011), but not for depression (Meades et al., 2011). Similar improvements in PTSD symptoms have been reported in previous reviews (Bastos et al., 2015; de Bruijn et al., 2020; de Graaff et al., 2018; Furuta et al., 2018; Sheen \& Slade, 2015) whereas no improvements were reported for anxiety, fear of childbirth (Bastos et al., 2015), and depression (Sheen \& Slade, 2015) at post-intervention. The greater improvement found in PTSD symptoms and fear of childbirth compared to anxiety and depression could be attributed to their different treatment modalities. PTSD resolves once the distress related to the specific traumatic event has been adequately addressed, and symptoms will not usually reoccur unless one encounters another traumatic event (Hollon, 2019). Fear of childbirth after a traumatic birth experience is a predictor of PTSD and therefore will reduce after an improvement in PTSD symptoms (Dikmen-Yildiz et al., 2018). On the other hand, depression and anxiety have been associated with self-doubt, making them notoriously difficult to treat successfully as any general setbacks in life can trigger a relapse (Buckman et al., 2018; Hollon, 2019; Rubin, 2020). Future interventions should address issues related to self-doubt, as well as support mothers to find ways to effectively manage life's stressors in order to alleviate anxiety and depression.

Anxiety and fear of childbirth showed similar improvements at 3-8 weeks follow-up and 2.5-5 months followup, respectively, compared to their immediate post-intervention scores. On the other hand, depression reported greater improvement at 3-8 weeks follow-up compared to their immediate post-intervention scores. PTSD symptoms reported less improvement at follow-up of longer than 2 months than below 2 months. As the longest follow-up duration was 5 months post-intervention, conducted by Ryding et al. (1998), future trials would need to extend the follow-up duration to examine the sustainability of intervention effect on postnatal mother's mental well-being in the long run. The larger improvement in depression reported at follow-up suggests that postnatal mothers may require more time to improve their depression. Previous reviews have reported similar findings of a lack of improvement in depression at post-intervention (Sheen \& Slade, 2015) but observed improvement at 2-5 months follow-up (Bastos et al., 2015). During the first few weeks after birth, mothers face numerous challenges while caring for their newborn, which could potentially challenge their self-worth and trigger depression (Hollon, 2019). Hence, more noticeable improvement at follow-up can be attributed to women harboring less self-doubt as they become more confident after months of motherhood.

Lesser improvements in PTSD symptoms over time could be due to some participants developing delayedonset PTSD. A longitudinal study reported that $5.8 \%$ of women who have experienced traumatic childbirth showed recovery at 4-6 weeks postpartum (did not meet the criteria for PTSD) but then proceeded to develop worse symptoms over time and met the criteria for PTSD at 6 months postpartum (Dikmen-Yildiz et al., 2018). Therefore, postnatal psychological support should be made available for at least 6 months postpartum in order to appropriately support mothers who have experienced traumatic childbirth as they are at risk of developing PTSD until then. Collaboration between various stakeholders such as policy makers, researchers, and healthcare institutions would be required to implement extensive and extended support for these vulnerable women.

Evidence suggests that psychological interventions delivered with or without the use of technology are able to improve the mental well-being of mothers after a traumatic birth experience. The technology used by the studies in this review include playing “Tetris” game on the Nintendo DS hand-held gaming console (Horsch et al., 2017) and online trauma-focused CBT modules (Nieminen et al., 2016). Technology provides participants the flexibility to choose when they would like to participate in the intervention, although Horsch et al. (2017) stipulated that they should play the game within the first 6 hours after birth. In Nieminen et al. (2016), mothers could attend the online courses at their convenience. Although most women found the intervention beneficial, a few preferred to consult a therapist (Nieminen et al., 2016), hence future trials could consider arranging online private therapy sessions for mothers upon request. 
Considering that mothers would be busy caring for their newborn, postnatal psychological interventions should promote flexibility and convenience (Slade, West, et al., 2020). Besides online interventions, other methods that could be considered include home visits (Kershaw et al., 2005) and organizing interventions on the dates of their follow-up appointments at the hospital or clinic.

All the three types of psychological interventions included in the meta-analysis (debriefing/counseling, CBT, and visuospatial cognitive task) demonstrated the potential to improve anxiety and PTSD symptoms in mothers who have experienced traumatic childbirth. However, only the debriefing/counseling subgroup reported improvement in depression but not the CBT and visuospatial cognitive task subgroups. This could be due to depression requiring more time to improve as a larger decrease at follow-up compared to immediate post-intervention was reported. On the other hand, fear of childbirth showed improvement via the use of debriefing/counseling methods (from meta-analyses) and mindfulness-based therapy (narrative synthesis); no data were available for other methods. However, as this review's subgroup analyses according to the type of psychological intervention were limited by the lack of studies in each subgroup (only one of two in some subgroups), the comparative effectiveness of each psychological intervention type on anxiety, depression, fear of childbirth, and PTSD symptoms could not be determined using the current evidence-a sentiment echoed by previous reviews (de Bruijn et al., 2020; de Graaff et al., 2018; Slade, Molyneux, et al., 2020). Furthermore, other promising types of psychological intervention for postnatal mothers such as expressive writing ( Qian et al., 2020), and eye movement desensitization and reprocessing treatment (Sandstrom et al., 2008; Stramrood et al., 2012), which were not examined in this review should be explored in future trials to examine their effects in women who have experienced traumatic childbirth.

Evidence from subgroup analysis suggests that indicated interventions are better than selective interventions at improving the mental well-being of women who have experienced traumatic childbirth. Previous reviews have reported that indicated interventions can alleviate psychological distress more effectively (Sheen \& Slade, 2015) and reduce medical costs (de Graaff et al., 2018; Lapp et al., 2010). According to the PTSD guidelines by the National Institute for Health and Clinical Excellence, conducting programs for adults pre-screened for PTSD symptoms is recommended instead of delivering programs to anyone who had undergone a specific traumatic event (National Collaborating Centre for Mental Health, 2005). This recommendation bears merit as everyone perceives trauma differently; some women reported in the study by Kershaw et al. (2005) did not attend the offered intervention because they were not traumatized by their childbirth experience even though undergoing operative birth methods is a risk factor for traumatic birth. Therefore, in order to examine the effect of psychological interventions on the mental well-being of women who have experienced traumatic childbirth more accurately, future trials should pre-screen all postnatal women for post-traumatic symptoms before recruiting them since any type of birth method can be perceived as traumatic (de Graaff et al., 2018). Pre-screening can be conducted using a standard scale such as the criterion A of the Diagnostic and Statistical Manual5 for PTSD to ensure consistency.

Only one included study conducted group-based intervention (Pour-Edalati et al., 2018), while the remaining studies conducted one-on-one interventions. One-on-one interventions allowed mothers to receive personalized support which they found desirable (Slade, Molyneux, et al., 2020). On the other hand, group-based interventions helped mothers to incorporate new knowledge more effectively and allowed them to share common experiences with their peers (Dennis, 2010; Teaford et al., 2018). Therefore, future trials could incorporate a mixture of both components for postnatal women to benefit from various sources of support.

\section{Implications for Future Research and Practice}

Future trials should be conducted in other geographical regions such as Asia, Africa, and America to understand the mental well-being of parents from other cultures who have experienced traumatic childbirth; cultural factors have been shown to affect one's childbirth experience (Jungari \& Paswan, 2020; Nomaguchi \& House, 2013). Moreover, future trials should actively engage and include fathers in postnatal psychological support by conducting joint sessions for parents and/or planning specialized sessions for fathers. Future interventions should also focus on helping mothers to manage their self-doubt and equip them with the necessary skills to face future adversities with the right mindset to prevent anxiety and depression. To promote flexibility and convenience for busy mothers of newborns, the use of technology, home visits, and planning interventions at postnatal follow-up appointments could be considered. As postnatal women can benefit from personalized professional support and through sharing experiences and advice with peers, future interventions could incorporate both elements. Since a previous study reported that peer support from mothers who recovered from postpartum depression can improve the emotional well-being of mothers who are at risk of postpartum depression (Shorey \& Ng, 2019), similar interventions could be planned for mothers who have experienced traumatic childbirth. To determine the comparative effectiveness of each type of psychological intervention on the various outcomes, future CCTs and RCTs should aim to examine anxiety, depression, fear of childbirth, and PTSD symptoms concurrently; no included study in this review assessed all the four outcomes. Longer term follow-up of participants beyond 5 months needs to be conducted to investigate the sustainability of the intervention effect on maternal mental well-being in the long run. Moreover, future trials should pre-screen all postnatal women for posttraumatic symptoms in order to more accurately identify those who have experienced traumatic childbirth so that 
indicated psychological interventions can be conducted for them. Due to the possibility of some women developing delayed-onset PTSD, and presenting few symptoms at 4-6 weeks postpartum that progress to significant symptoms at 6 months postpartum, extended support for mothers who have experienced traumatic childbirth could be implemented through collaboration among stakeholders and healthcare institutions. Lastly, as compounding factors such as intimate partner violence and experience of childhood sexual abuse have been associated with future traumatic birth experience (Oliveira et al., 2017), future studies could examine the presence of these factors among their participants to isolate the impact of prior trauma on childbirth trauma.

\section{Limitations}

The following limitations may affect the accuracy of this review's findings. Relevant studies may have been missed out due to unclear titles or abstracts and poor indexing. Review outcomes were analyzed by studies using the self-report method thus it may have contributed to social desirability bias. As the extraction of only end-point data was performed for meta-analysis, disparities between the baseline scores of both intervention and control groups were not considered. Due to the limited number of overall included studies (only eight included studies and six included in meta-analysis) and a number of studies which did not assess outcomes for anxiety, depression, fear of childbirth, and PTSD symptoms, some subgroup analysis had only one or two studies in each subgroup and this could have affected the results' reliability and validity. Since the small number of studies ultimately included in this review only focused on European and Middle Eastern regions, the findings could not be generalized to other parts of the world. More notably, this review's findings have a lack of relevance to North American cultures, as well as their various subcultural and socioeconomic groups. Furthermore, the included studies also failed to consider prior trauma that these women may have experienced such as intimate partner violence and childhood sexual abuse that could contribute to their development of PTSD after childbirth. Lastly, as review outcomes were rated as either low or very low quality via the GRADE approach, there is a limited to very little confidence in the effect size estimated (Higgins \& Green, 2011) in this review.

\section{Conclusion}

This review found that psychological interventions were more effective in decreasing fear of childbirth and improving PTSD symptoms compared to anxiety and depression among mothers who have experienced traumatic childbirth. Depression showed greater improvement at 3-8 weeks follow-up compared to immediate post-intervention. Improving anxiety and depression is challenging, hence interventions should educate mothers to face setbacks with the right mindset to prevent relapse. Future trials should involve fathers, deliver personalized treatment and group-based support to mothers, and prioritize on developing flexible and convenient interventions. Indicated interventions should be advocated and closer examination of all the available promising psychological interventions is needed to assess each type's comparative effectiveness. Longer follow-up durations would also be needed to examine the long-term sustainability of intervention effect. Due to the phenomenon of delayed-onset PTSD, stakeholders and healthcare institutions could work together to provide relevant and extensive psychological support for mothers who have experienced traumatic childbirth. Lastly, considering the very low to low quality of evidence using the GRADE approach in this review, future research is needed to validate current findings.

\section{Acknowledgments}

It is Aetion Chair, SD is management committee member MK and SS are members of the network. Shefaly_Shorey. and Maaike Fobelets are members of working group 3.3. Soo Downe is a management committee member and Joan Lalor is the Action Chair of the EU COST Action 18211: DEVoTION: Perinatal Mental Health and Birth-Related Trauma: Maximising best practice and optimal outcomes supported by COST (European Cooperation in Science and Technology). The authors would like to thank the National University Health System, Research Support Unit, for assistance in the language editing of this manuscript.

\section{ORCID iDs}

Shefaly Shorey https://orcid.org/0000-0001-5583-2814

Soo Downe https://orcid.org/0000-0003-2848-2550

Sofia O Byrne https://orcid.org/0000-0002-5197-0467

\section{Author Biographies}

Shefaly Shorey, $\mathrm{PhD}$, is an assistant professor at Alice Lee Centre for Nursing Studies, National University of Singapore. Her research interests lie in maternal and children health.

Soo Downe, PhD, is a professor of Midwifery studies at University of Central Lancashire. Her research interests lie in maternity care and childbirth. 
Joelle Yan Chua Xin, BSc. (Hons), is a research assistant in the Alice Lee Centre for Nursing Studies at National

University of Singapore. Her research focuses on the general improvement of mental well-being.

Sofia O Byrne is a PhD candidate at Trinity College Dublin. Her research interests lie in maternal health and maternity care.

Maaike Fobelets, PhD, is a post-doctoral researcher at Faculty of Medicine and Pharmacy, Department of Public Health Sciences, Biostatistics and Medical Informatics (BISI) Research Group, Vrije Universiteit Brussel. Her research interests lie in midwifery, childbirth, and maternity care.

Joan Lalor, PhD, is a professor in Midwifery, School of Nursing \& Midwifery, Trinity College Dublin. Her research interests lie in maternity care and women's health.

\section{References}

Abdollahpour, S., Keramat, A., Mousavi, S. A., Khosravi, A., \& Motaghi, Z. (2018). The effect of debriefing and brief cognitive-behavioral therapy on postpartum depression in traumatic childbirth: A randomized clinical trial. Journal of Midwifery \& Reproductive Health, 6(1), 1122-1131. https://doi.org/10.22038/jwrh.2017.1000 $\underline{0}$.

Q1 Agius, A., Xuereb, R. B., Carrick-Sen, D., Sultana, R., \& Rankin, J. (2016). The co-existence of depression, anxiety and post-traumatic stress symptoms in the perinatal period: A systematic review. Midwifery, 36, 7079. https://doi.org/10.1016/j.midw.2016.02.013.

Alcorn, K. L., O’Donovan, A., Patrick, J. C., Creedy, D., \& Devilly, G. J. (2010). A prospective longitudinal study of the prevalence of post-traumatic stress disorder resulting from childbirth events. Psychological Medicine, 40(11), 1849-1859. https://doi.org/10.1017/S0033291709992224.

Asadzadeh, L., Jafari, E., Kharaghani, R., \& Taremian, F. (2020). Effectiveness of midwife-led brief counseling intervention on post-traumatic stress disorder, depression, and anxiety symptoms of women experiencing a traumatic childbirth: a randomized controlled trial. BMC Pregnancy and Childbirth, 20(1), 142. https://doi.org/10.1186/s12884-020-2826-1.

Ayers, S., Claypool, J., \& Eagle, A. (2006). What happens after a difficult birth? Postnatal debriefing services. British Journal of Midwifery, 14(3), 157-161. https://doi.org/10.12968/bjom.2006.14.3.20577.

Baer, R. A. (2003). Mindfulness training as a clinical intervention: A conceptual and empirical review. Clinical Psychology (New York, N.Y.), 10(2), 125-143. https://doi.org/10.1093/clipsy.bpgo $\underline{015}$.

Q2 Bastos, M. H., Furuta, M., Small, R., McKenzie-McHarg, K., \& Bick, D. (2015). Debriefing interventions for the prevention of psychological trauma in women following childbirth. [The Cochrane Database of Systematic Reviews Electronic Resource], (4), Cd007194. https://doi.org/10.1002/14651858.CD007194.pub2.

Bauer, A., Parsonage, M., Knapp, M., Iemmi, V., \& Adelaja, B. (2014). Costs of perinatal mental health problems. London School of Economics and Political Science.

Bavelier, D., \& Green, C. S. (2003). Action video game modifies visual selective attention. Nature, 423(6939), 534-537. https://doi.org/10.1038/nature01647.

Beck, C. T. (2016). Posttraumatic stress disorder after birth:Aa metaphor analysis. $M C N$, the American journal of maternal child nursing, 41(2), 76-83. https://doi.org/10.1097/NMC.0000000000000211.

Blasio, P. D., Ionio, C., \& Confalonieri, E. (2009). Symptoms of postpartum PTSD and expressive writing: A prospective study. Journal of Prenatal \& Perinatal Psychology \& Health, 24(1), 49.

Blom, E. A., Jansen, P. W., Verhulst, F. C., Hofman, A., Raat, H., Jaddoe, V. W. V., Coolman, M., Steegers, E. A. P., \& Tiemeier, H. (2010). Perinatal complications increase the risk of postpartum depression. The generation R study: Perinatal risk factors of postpartum depression. BJOG : an International Journal of Obstetrics and Gynaecology, 117(11), 1390-1398. https://doi.org/10.1111/j.1471-0528.2010.02660.x.

Borg Cunen, N., McNeill, J., \& Murray, K. (2014). A systematic review of midwife-led interventions to address post partum post-traumatic stress. Midwifery, 30(2), 170-184. https://doi.org/10.1016/j. 
Bryant, R. A. (2007). Early intervention for post-traumatic stress disorder. Early Intervention in Psychiatry, 1(1), 19-26. https://doi.org/10.1111/j.1751-7893.2007.00006.x.

Buckman, J. E. J., Underwood, A., Clarke, K., Saunders, R., Hollon, S. D., Fearon, P., \& Pilling, S. (2018). Risk factors for relapse and recurrence of depression in adults and how they operate: A four-phase systematic review and meta-synthesis. Clinical Psychology Review, 64, 13-38. https://doi.org/https://doi.org/10.1016/j.cp r.2018.07.005.

Cava-Tadik, Y., Brown, G. L., \& Mangelsdorf, S. C. (2020). Fathers' satisfaction with physical affection before and after the birth of a new baby: Cross-parent effects and associations with family dynamics. Journal of Family Issues, 41(4), 415-436. https://doi.org/10.1177/0192513X19875779.

de Bruijn, L., Stramrood, C. A., Lambregtse-van den Berg, M. P., \& Rius Ottenheim, N. (2020). Treatment of posttraumatic stress disorder following childbirth. Journal of Psychosomatic Obstetrics and Gynaecology, 41(1), 5-14. https://doi.org/10.1080/0167482x.2019.1593961.

de Graaff, L. F., Honig, A., van Pampus, M. G., \& Stramrood, C. A. I. (2018). Preventing post-traumatic stress disorder following childbirth and traumatic birth experiences: A systematic review. Acta Obstetricia et Gynecologica Scandinavica, 97(6), 648-656. https://doi.org/10.1111/aogs.13291.

Dennis, C.-L. (2010). Postpartum depression peer support: Maternal perceptions from a randomized controlled trial. Int J Nurs Stud, 47(5), 560-568. https://doi.org/https://doi.org/10.1016/j.jinurstu.2009.10.015.

DEVOTION CA18211. (2020). Birth trauma Research is a necessity. DEVOTION CA18211. https://www.c a18211.eu/why-this-research/ (accessed 22 February 2021).

Dikmen-Yildiz, P., Ayers, S., \& Phillips, L. (2017). Depression, anxiety, PTSD and comorbidity in perinatal women in Turkey: A longitudinal population-based study. Midwifery, 55, 29-37. https://doi.org/10.1016/j. w.2017.09.001.

Dikmen-Yildiz, P., Ayers, S., \& Phillips, L. (2018). Longitudinal trajectories of post-traumatic stress disorder (PTSD) after birth and associated risk factors. $J$ Affect Disord, 229, 377-385. https://doi.org/10.1016/j.jad.201 7.12.074.

D’Souza, S., D’Souza, S., Waldie, K. E., Waldie, K. E., Peterson, E. R., Peterson, E. R., Underwood, L., Underwood, L., Morton, S. M. B., \& Morton, S. M. B. (2019). Antenatal and postnatal determinants of behavioural difficulties in early childhood: Evidence from growing up in New Zealand. Child Psychiatry and Human Development, 50(1), 45-60. https://doi.org/10.1007/s10578-018-0816-6.

Eccleston C., Fisher E., Law E., Bartlett J., and Palermo T. M. (2015). Psychological interventions for parents of children and adolescents with chronic illness. Cochrane Database of Systematic Reviews. Advance online publication. https://doi.org/10.1002/14651858.CD009660.pub3

Egger, M., \& Smith, G. D. (1997). Meta-analysis: Potentials and promise. Bmj: British Medical Journal, 315(7119), 1371. https://doi.org/10.1136/bmj.315.7119.1371.

Eide, K. T., Morken, N.-H., \& Bærøe, K. (2019). Maternal reasons for requesting planned cesarean section in Norway: a qualitative study. BMC Pregnancy and Childbirth, 19(1), 102. https://doi.org/10.1186/s12884-019 $\underline{-2250-6 .}$.

Fairbrother, N., Thordarson, D. S., \& Stoll, K. (2018). Fine tuning fear of childbirth: the relationship between childbirth fear questionnaire subscales and demographic and reproductive variables. Journal of Reproductive and Infant Psychology, 36(1), 15-29. https://doi.org/10.1080/02646838.2017.1396300.

Furuta, M., Horsch, A., Ng, E. S. W., Bick, D., Spain, D., \& Sin, J. (2018). Effectiveness of trauma-focused psychological therapies for treating post-traumatic stress disorder symptoms in women following childbirth: A systematic review and meta-analysis. Front Psychiatry, 9, 591. https://doi.org/10.3389/fpsyt.2018.00591.

Furuta, M., Sandall, J., \& Bick, D. (2012). A systematic review of the relationship between severe maternal morbidity and post-traumatic stress disorder. BMC Pregnancy and Childbirth, 12, 125. https://doi.org/10.118 
Gamble, J. A., Creedy, D. K., Webster, J., \& Moyle, W. (2002). A review of the literature on debriefing or non-directive counselling to prevent postpartum emotional distress. Midwifery, 18(1), 72-79. https://doi.org/1 $\underline{0.1054 / \text { midw.2001.0287. }}$.

Giallo, R., Bahreinian, S., Brown, S., Cooklin, A., Kingston, D., \& Kozyrskyj, A. (2015). Maternal depressive symptoms across early childhood and asthma in school children: findings from a Longitudinal Australian Population Based Study. Plos One, 10(3), e0121459. https://doi.org/10.1371/journal.pone.012145 $\underline{9}$.

Glasheen, C., Glasheen, C., Richardson, G. A., Richardson, G. A., Fabio, A., \& Fabio, A. (2010). A systematic review of the effects of postnatal maternal anxiety on children. Archives of Women's Mental Health, 13(1), 61-74. https://doi.org/10.1007/s00737-009-0109-y.

GRADEpro. (2015). GRADEpro. https://gradepro.org.

Grekin, R., \& O’Hara, M. W. (2014). Prevalence and risk factors of postpartum posttraumatic stress disorder: A meta-analysis. Clinical Psychology Review, 34(5), 389-401. https://doi.org/10.1016/j.cpr.2014.05.003.

Higgins, J. P., \& Green, S. (2011). Cochrane handbook for systematic reviews of interventions. John Wiley \& Sons.

Hollon, S. D. (2019). Treatment of Depression Versus Treatment of PTSD. American Journal of Psychiatry, 176(4), 259-261. https://doi.org/10.1176/appi.ajp.2019.19020173.

Holmes, E. A., James, E. L., Coode-Bate, T., \& Deeprose, C. (2009). Can playing the computer game "Tetris" reduce the build-up of flashbacks for trauma? A proposal from cognitive science. Plos One, 4(1), e4153. https://doi.org/10.1371/journal.pone.0004153.

Horsch, A., Schobinger, E., \& Stuijfzand, S. (2020). Acute and Post-traumatic Stress Disorder Symptoms in Mothers and Fathers Following Childbirth: A Prospective Cohort Study.(Clinical report). Front Psychiatry, 11, 562054. https://doi.org/10.3389/fpsyt.2020.562054.

Horsch, A., Vial, Y., Favrod, C., Harari, M. M., Blackwell, S. E., Watson, P., Iyadurai, L., Bonsall, M. B., \& Holmes, E. A. (2017). Reducing intrusive traumatic memories after emergency caesarean section: A proof-ofprinciple randomized controlled study. Behaviour Research and Therapy, 94, 36-47. https://doi.org/10.1016/j. brat.2017.03.018.

Inglis, C., Sharman, R., \& Reed, R. (2016). Paternal mental health following perceived traumatic childbirth. Midwifery, 41, 125-131. https://doi.org/10.1016/j.

Jungari, S., \& Paswan, B. (2020). Supported motherhood? An examination of the cultural context of male participation in maternal health care among tribal communities in India. Journal of Biosocial Science, 52(3), 452-471. https://doi.org/10.1017/S0021932019000580.

Kempler, L., Sharpe, L., Miller, C. B., \& Bartlett, D. J. (2016). Do psychosocial sleep interventions improve infant sleep or maternal mood in the postnatal period? A systematic review and meta-analysis of randomised controlled trials. Sleep Medicine Reviews, 29, 15-22. https://doi.org/10.1016/j.smrv.2015.08.002.

Kershaw, K., Jolly, J., Bhabra, K., \& Ford, J. (2005). Randomised controlled trial of community debriefing following operative delivery. BJOG: an International Journal of Obstetrics and Gynaecology, 112(11), 1504-1509. https://doi.org/10.1111/j.1471-0528.2005.00723.x.

Lapp, L. K., Agbokou, C., Peretti, C. S., \& Ferreri, F. (2010). Management of post traumatic stress disorder after childbirth: a review. Journal of Psychosomatic Obstetrics and Gynaecology, 31(3), 113-122. https://doi. org $10.3109 / 0167482 x .2010 .503330$.

Leach, L. S., Poyser, C., \& Fairweather-Schmidt, K. (2017). Maternal perinatal anxiety: A review of prevalence and correlates. Clinical Psychologist (Australian Psychological Society), 21(1), 4-19. https://doi.or $\mathrm{g} / \underline{10.1111 / \mathrm{cp} .12058 .}$. 
Luo, D., Wan, X., Liu, J., \& Tong, T. (2018). Optimally estimating the sample mean from the sample size, median, mid-range, and/or mid-quartile range. Statistical Methods in Medical Research, 27(6), 1785-1805. htt ps://doi.org/10.1177/0962280216669183.

Meades, R., Pond, C., Ayers, S., \& Warren, F. (2011). Postnatal debriefing: Have we thrown the baby out with the bath water? Behaviour Research and Therapy, 49(5), 367-372. https://doi.org/10.1016/j.brat.2011.0 3.002 .

Moher, D., Liberati, A., Tetzlaff, J., \& Altman, D. G. (2009). Preferred reporting items for systematic reviews and meta-analyses: The PRISMA statement. Annals of Internal Medicine, 151(4), 264-269. https://doi.org/10. $\underline{1136 / \mathrm{bmj} . \mathrm{b} 2535 .}$.

National Collaborating Centre for Mental Health. (2005). National Institute for health and clinical excellence: Guidance (Post-traumatic stress disorder: The management of PTSD in adults and children in primary and secondary care. Gaskell Copyright (C) 2005, The Royal College of Psychiatrists \& The British Psychological Society.

Nieminen, K., Berg, I., Frankenstein, K., Viita, L., Larsson, K., Persson, U., Spånberger, L., Wretman, A., Silfvernagel, K., Andersson, G., \& Wijma, K. (2016). Internet-provided cognitive behaviour therapy of posttraumatic stress symptoms following childbirth-a randomized controlled trial. Cognitive Behaviour Therapy, 45(4), 287-306. https://doi.org/10.1080/16506073.2016.1169626.

Nomaguchi, K., \& House, A. N. (2013). Racial-ethnic disparities in maternal parenting stress: The role of structural disadvantages and parenting values. Journal of Health and Social Behavior, 54(3), 386-404. https:// doi.org/10.1177/0022146513498511.

Oliveira, A. G. e. S. d., Reichenheim, M. E., Moraes, C. L., Howard, L. M., \& Lobato, G. (2017). Childhood sexual abuse, intimate partner violence during pregnancy, and posttraumatic stress symptoms following childbirth: a path analysis. Archives of Women's Mental Health, 20(2), 297-309. https://doi.org/10.1007/s0073 7-016-0705-6.

Peeler, S., Chung, M. C., Stedmon, J., \& Skirton, H. (2013). A review assessing the current treatment strategies for postnatal psychological morbidity with a focus on post-traumatic stress disorder. Midwifery, 29(4), 377-388. https://doi.org/10.1016/j. midw.2012.03.004.

Poh, H. L., Koh, S. S. L., \& He, H. G. (2014). An integrative review of fathers' experiences during pregnancy and childbirth. Int Nurs Rev, 61(4), 543-554. https://doi.org/10.1111/inr.12137.

Pour-Edalati, M., Moghadam, N. K., Shahesmaeili, A., \& Salehi-Nejad, P. (2018). Exploring the effect of mindfulness-based stress reduction on childbirth fear among single-child mothers in the city of Kerman, Iran (2017): A clinical trial study. Medical-Surgical Nursing Journal, 7(4), 1-7. https://doi.org/10.5812/msnj.9024 7.

Qian, J. L., Zhou, X. L., Sun, X. S., Wu, M. W., Sun, S. W., \& Yu, X. Y. (2020). Effects of expressive writing intervention for women's PTSD, depression, anxiety and stress related to pregnancy: A meta-analysis of randomized controlled trials. Psychiatry Research, 288, 293. https://doi.org/10.1016/j.psychres.2020.11293 $\underline{3}$.

Regier, D. A., Kuhl, E. A., \& Kupfer, D. J. (2013). The DSM-5: Classification and criteria changes. World Psychiatry, 12(2), 92-98. https://doi.org/10.1002/wps.20050.

Rowan, C., Bick, D., \& Bastos, M. H. (2007). Postnatal debriefing interventions to prevent maternal mental health problems after birth: exploring the gap between the evidence and UK policy and practice. Worldviews on Evidence-Based Nursing, 4(2), 97-105. https://doi.org/10.1111/j.1741-6787.2007.00088.x.

Rubin J. (2020). Anxiety Symptoms and self-doubt. Lost Highway Blues. https://janerubinphd.com/anxiety-sy. mptoms-and-self-doubt/ (accessed 26 January 2020).

Ryding, E. L., Wijma, K., \& Wijma, B. (1998). Postpartum counselling after an emergency cesarean. Clinical Psychology \& Psychotherapy, 5(4), 231-237. https://doi.org/10.1002/(sici) 1099-0879(199812) 5:4<231::Aid-c pp $172>3.0$. Co $; \underline{2-9}$. 
Sandstrom, M., Wiberg, B., Willman, A. K., Wikman, M., \& Hogberg, U. (2008). A pilot study of eye movement desensitisation and reprocessing treatment (EMDR) for post-traumatic stress after childbirth. Midwifery, 24(1), 62-73. https://doi.org/10.1016/j.midw.2006.07.008.

Sawilowsky, S. S. (2009). New effect size rules of thumb. Journal of Modern Applied Statistical Methods, 8(2), 597-599. https://doi.org/10.22237/jmasm/1257035100.

Sedgwick, P. (2013). Meta-analyses: heterogeneity and subgroup analysis. BMJ: British Medical Journal, 346, f4040. https://doi.org/10.1136/bmj.

Sheen, K., \& Slade, P. (2015). The efficacy of 'debriefing' after childbirth: Is there a case for targeted intervention? J Reprod Infant Psychol, 33(3), 308-320. https://doi.org/10.1080/02646838.2015.1009881.

Shi, J., Luo, D., Weng, H., Zeng, X.-T., Lin, L., Chu, H., \& Tong, T. (2020). Optimally estimating the sample standard deviation from the five-number summary. Research Synthesis Methods, 11(5), 641-654. https://doi.or $\mathrm{g} / \underline{10.1002}$ j.jsm.1429.

Shorey, S., Ang, L., Goh, E. C. L., \& Lopez, V. (2019). Paternal involvement of Singaporean fathers within six months postpartum: A follow-up qualitative study. Midwifery, 70, 7-14. https://doi.org/10.1016/j. $\underline{\text { midw.20 }}$ $\underline{18.12 .003 .}$.

Shorey, S., \& Ng, E. D. (2019). Evaluation of a technology-based peer-support intervention program for preventing postnatal depression (part 2): Qualitative study. Journal of Medical Internet Research, 21 (8), e12915. https://doi.org/10.2196/12915.

Shorey S. and Wong P. Z. E. (2020). Traumatic childbirth experiences of new parents: A meta-synthesis. Trauma, Violence, \& Abuse. Advance online publication. https://doi.org/10.1177/1524838020977161

Shorey, S., Yang, Y. Y., \& Ang, E. (2018). The impact of negative childbirth experience on future reproductive decisions: A quantitative systematic review. Journal of Advanced Nursing, 74(6), 1236-1244. htt ps://doi.org/10.1111/jan.13534.

Slade P., Molyneux D. R., and Watt D. A. (2020b). A systematic review of clinical effectiveness of psychological interventions to reduce post traumatic stress symptoms following childbirth and a meta-synthesis of facilitators and barriers to uptake of psychological care. Journal of Affective Disorders. Advance online publication. $\underline{\text { https://doi.org/10.1016/j.jad.2020.11.092 }}$

Slade P., West H., Thomson G., Lane S., Spiby H., Edwards R. T., Charles J. M., Garrett C., Flanagan B., Treadwell M., Hayden E., and Weeks A. (2020a). STRAWB2 (stress and wellbeing after childbirth): A randomised controlled trial of targeted self-help materials to prevent post-traumatic stress disorder following childbirth. BJOG : an International Journal of Obstetrics and Gynaecology, 127(7), 886-896. https://doi.org/1 $\underline{0.1111 / 1471-0528.16163}$

Söderquist, J., Wijma, K., \& Wijma, B. (2002). Traumatic stress after childbirth: the role of obstetric variables. Journal of Psychosomatic Obstetrics and Gynaecology, 23(1), 31-39. https://doi.org/10.3109/016748202090 $\underline{93413}$.

Söderquist, J., Wijma, B., \& Wijma, K. (2006). The longitudinal course of post-traumatic stress after childbirth. Journal of Psychosomatic Obstetrics and Gynaecology, 27(2), 113-119. https://doi.org/10.1080/01 $\underline{674820600712172 .}$.

Sterne, J. A., Sutton, A. J., Ioannidis, J. P., Terrin, N., Jones, D. R., Lau, J., Carpenter, J., Rücker, G., Harbord, R. M., \& Schmid, C. H. (2011). Recommendations for examining and interpreting funnel plot asymmetry in meta-analyses of randomised controlled trials. Bmj: British Medical Journal, 343, d4002. http s://doi.org/10.1136/bmj.

Storksen, H. T., Eberhard-Gran, M., Garthus-Niegel, S., \& Eskild, A. (2012). Fear of childbirth; the relation to anxiety and depression. Acta Obstetricia et Gynecologica Scandinavica, 91(2), 237-242. https://doi.org/10.11 $\underline{11 / \mathrm{j} .1600-0412.2011 .01323 . \mathrm{x} .}$

Stramrood, C. A. I., van der Velde, J., Doornbos, B., Marieke Paarlberg, K., Weijmar Schultz, W. C. M., \& van Pampus, M. G. (2012). The patient observer: Eye-movement desensitization and reprocessing for the 
Teaford, D., McNiesh, S., \& Goyal, D. (2018). New mothers' experiences with online postpartum forums. MCN, the American journal of maternal child nursing, 44(1), 40-45. https://doi.org/10.1097/NMC.00000000 $\underline{00000489 .}$.

\title{
Notes
}

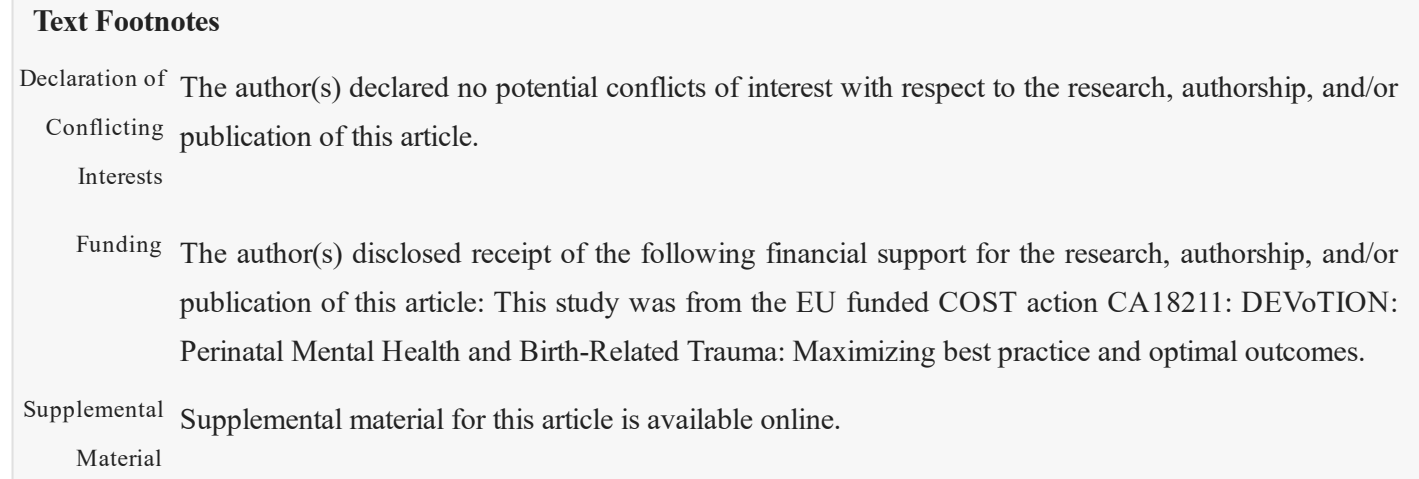

\section{Supplemental Material}

\author{
sj-pdf-1-tva-10.1177 15248380211060808.pdf
}

sj-pdf-1-tva-10.1177_15248380211060808 - Supplemental Material for Effectiveness of Psychological Interventions to Improve the Mental Well-Being of Parents Who Have Experienced Traumatic Childbirth: A Systematic Review and Meta-Analysis

Supplemental Material, sj-pdf-1-tva-10.1177_15248380211060808 for Effectiveness of Psychological Interventions to Improve the Mental Well-Being of Parents Who Have Experienced Traumatic Childbirth: A Systematic Review and Meta-Analysis by Shefaly Shorey, Soo Downe, Joelle Yan Xin Chua, Sofia O. Byrne, Maaike Fobelets and Joan Lalor in Trauma, Violence, \& Abuse

\section{Supplemental Material}

sj-pdf-2-tva-10.1177_15248380211060808.pdf

sj-pdf-2-tva-10.1177_15248380211060808 - Supplemental Material for Effectiveness of Psychological Interventions to Improve the Mental Well-Being of Parents Who Have Experienced Traumatic Childbirth: A Systematic Review and Meta-Analysis

Supplemental Material, sj-pdf-2-tva-10.1177_15248380211060808 for Effectiveness of Psychological Interventions to Improve the Mental Well-Being of Parents Who Have Experienced Traumatic Childbirth: A Systematic Review and Meta-Analysis by Shefaly Shorey, Soo Downe, Joelle Yan Xin Chua, Sofia O. Byrne, Maaike Fobelets and Joan Lalor in Trauma, Violence, \& Abuse

\section{Queries and Answers}

\section{Q1}

Query: Agius et al. (2016); Buckman et al. (2018); Dikmen-Yildiz et al. (2017); Please provide issue number for refs. DikmenYildiz et al. (2018); Furuta et al. (2018); Horsch et al. (2020); Inglis et al. (2016); Kempler et al. (2016); Qian et al. (2020); Sedgwick (2013); Shorey et al. (2019); Sterne et al. (2011)

Answer: Dear editors,

We are unable to retrieve the issue numbers for the references that were indicated. Nonetheless, we have ensured that their doi were provided to identify all materials cited in this manuscript. 
Query: Please provide volume number for ref. Bastos et al. (2015).

Answer: Dear editors,

The reference for Bastos et al. (2015) is published as a cochrane review, hence no volume number is provided. 